CHAPITRE 4

\title{
Interaction laser-plasma et laser petawatt
}

ARNOLD MIGUS ET DENIS PESME 



\section{Interaction laser-plasma}

\subsection{Physique mise en jeu}

La physique de l'interaction laser-plasma couple trois catégories de phénomènes entre eux :

- des processus de type "hydrodynamique », caractérisés par des échelles spatiales de l'ordre du mm et des échelles de temps de l'ordre de la nanoseconde;

- des processus de type "optique non linéaire », que sont les instabilités paramétriques, caractérisés par des échelles spatiales de l'ordre de quelques $\mu \mathrm{m}$ et des échelles de temps de l'ordre d'une fraction de picoseconde;

- ces deux premières catégories de processus sont décrites à l'aide d'équations dites de type "fluide", au sens où ils sont décrits par des équations aux dérivées partielles dont les seules variables sont le temps et l'espace ;

- des processus de type "cinétique », propres à la physique des plasmas parce que mettant en jeu des mécanismes d'interaction ondes-particules, caractérisés par des échelles spatiales submicrométriques (la longueur de Debye) et des échelles de temps de l'ordre de quelques femtosecondes (l'inverse de la fréquence plasma électronique). Cette troisième classe de processus nécessite une description de type " cinétique » (du type équation de Vlasov pour une description analytique, et à l'aide de codes PIC pour la simulation numérique), dans laquelle les variables sont les positions, les vitesses et le temps.

Présentons succinctement de quelle façon ces trois catégories de processus sont intimement liées entre elles, en commençant par les mécanismes à la base de I'interaction laser-plasma, les instabilités paramétriques. Dans les deux schémas de fusion par laser - attaque directe ou indirecte - les faisceaux laser doivent se propager dans des plasmas sous denses de grandes dimensions (plusieurs milliers de longueurs d'onde des faisceaux laser) avant de déposer leur énergie au voisinage de la densité critique. Les valeurs très élevées des flux de lumière quasi cohérente (la cohérence des faisceaux laser ne pouvant qu'être partiellement réduite) sont très favorables à l'excitation d'instabilités paramétriques analogues à celles rencontrées en optique non linéaire. Comme dans ce domaine, elles peuvent être de deux types:

- les unes sont liées à la non-linéarité de l'indice et provoquent essentiellement une redistribution de l'intensité du faisceau par filamentation ou 
autofocalisation de la lumière : ces mécanismes sont de nature semblable à ceux provoqués par l'effet Kerr; ces instabilités peuvent provoquer la déflexion et l'éclatement des faisceaux laser, ce qui perturbe en particulier la position des zones de dépôt d'énergie sur la paroi du holraüm dans le cas de l'attaque indirecte;

- les autres peuvent être considérées comme une diffusion du rayonnement sur les fluctuations de densité générées par des ondes devenues instables en présence du rayonnement laser. Dans cette deuxième catégorie, deux instabilités jouent un rôle particulier :

- la diffusion Raman stimulée, correspondant à l'excitation d'ondes de densité électroniques, de fréquence proche de la fréquence plasma,

- la diffusion Brillouin stimulée, mettant en jeu des ondes acoustiques ioniques de fréquence beaucoup plus basse.

Ces deux instabilités peuvent provoquer la rétrodiffusion des faisceaux laser incidents, ce qui correspond à la perte d'une fraction de l'énergie laser.

Les conditions de développement linéaire de ces instabilités s'obtiennent à partir du facteur de gain linéaire. Ce dernier représente la prédiction d'ordre zéro du niveau de développement de ces instabilités, et il dépend fortement des paramètres hydrodynamiques du plasma : les valeurs locales des densités électroniques, des températures électroniques et ioniques, $d$ 'une part, et, d'autre part, la taille des gradients spatiaux de ces quantités ainsi que du flot d'écoulement. C'est de cette façon que les instabilités paramétriques sont couplées avec l'hydrodynamique du plasma, l'hydrodynamique étant elle-même couplée en retour avec l'interaction laser plasma via les mécanismes d'absorption par le plasma de l'énergie des faisceaux laser et via le dépôt de moment induit par les différents processus de diffusion des faisceaux. Il existe donc une première boucle de rétroaction non linéaire : hydrodynamique $\leftrightarrow$ instabilités paramétriques.

Par ailleurs, ces instabilités paramétriques, que l'on peut donc décrire à l'ordre le plus bas de façon "fluide», sont en mesure d'induire des effets de type « cinétique »: les ondes longitudinales générées par le développement de ces instabilités peuvent en effet atteindre des amplitudes suffisamment élevées pour piéger des particules du plasma et mener ainsi à des fonctions de distribution éloignées de l'équilibre. Ces effets cinétiques ont plusieurs conséquences : $d^{\prime}$ une part ils peuvent donner naissance à des populations électroniques très énergétiques, qui sont en mesure de préchauffer la cible et, de là, réduire sa compression (un tel préchauffage est l'un des risques majeurs de l'instabilité de diffusion Raman stimulée); d'autre part, ils peuvent modifier la valeur de l'amortissement des ondes mises en jeu et donner lieu à des effets de décalage 
de fréquence non linéaire, ce qui a des conséquences immédiates sur l'évolution non linéaire des instabilités paramétriques. Il existe donc une deuxième boucle de rétroaction non linéaire : instabilités paramétriques $\leftrightarrow$ effets cinétiques.

D'une façon générale, les différents processus mis en jeu dans l'interaction laser-plasma sont couplés non linéairement entre eux, et leur couplage peut être non local en espace, ce qui a d'importantes conséquences pour ce qui concerne leur simulation numérique. En effet, le couplage entre les instabilités de filamentation et l'instabilité de diffusion Brillouin vers l'avant peut donner lieu, dans le cas de faisceaux lissés spatialement, à ce que l'on appelle « le lissage induit par le plasma », par lequel le faisceau devient plus incohérent en temps et en espace au cours de sa propagation dans le plasma. Ainsi, si ce lissage induit par le plasma se produit du côté entrant de l'onde laser dans le plasma, il est en mesure de modifier significativement l'interaction laser-plasma plus en avant dans la propagation des ondes incidentes. On ne peut donc pas se contenter $d^{\prime}$ analyses locales simples des mécanismes d'interaction laser-plasma.

En conclusion, I'interaction laser-plasma met directement en jeu toute la physique non linéaire des plasmas chauds, caractérisée par des processus de nature très différente (hydrodynamique, optique non linéaire, effets cinétiques), et des échelles spatiales et temporelles s'étalant sur plus de trois ordres de grandeur. Ces différents processus sont couplés entre eux par des boucles de rétroaction non linéaire, les couplages pouvant ne pas être locaux spatialement. De plus, ces différentes instabilités peuvent être présentes simultanément et interagir entre elles, ce qui rend considérablement difficile la prédiction de leur évolution non linéaire.

Mentionnons ici une autre caractéristique très importante de l'interaction laser-plasma : le caractère partiellement aléatoire du champ transverse caractérisant les faisceaux laser au sein du plasma, ces faisceaux ayant été volontairement rendus partiellement incohérents. Au point de vue expérimental, les résultats concernant l'interaction laser-plasma ont en effet longtemps été obscurcis par la mauvaise qualité optique des faisceaux. Aujourd'hui, le lissage optique (réalisé à l'aide de lames de phases aléatoires, éventuellement combiné à une incohérence temporelle des faisceaux laser) permet de disposer de faisceaux dont les caractéristiques sont mieux connues au prix d'une plus grande complexité, puisque phases et amplitudes sont alors des quantités fluctuantes en temps et en espace. L'une des caractéristiques essentielles des faisceaux lissés est qu'ils comportent de nombreux maxima locaux d'intensité dans le volume où ils sont focalisés : ces « points chauds » sont appelés des " grains de tavelure » (speckles en anglais) dans le contexte des faisceaux lissés. Les dimensions spatiales de ces grains de tavelure sont déterminées par les caractéristiques des lentilles de focalisation. Ils sont répartis de façon aléatoire en espace, et leur intensité, elle aussi aléatoire, est répartie selon une loi de distribution qui est connue (loi 
de Rayleigh). Ainsi on peut considérer, dans une première approche, qu'un faisceau laser incident est constitué d'un " fond » moyen, dont l'intensité est de l'ordre de l'intensité moyenne, et de grains de tavelure dont l'intensité peut être plusieurs fois l'intensité moyenne (pour les plasmas actuels de petite dimension, l'intensité du grain de tavelure le plus intense est typiquement dix fois l'intensité moyenne).

Signalons enfin que l'importance des enjeux de l'interaction laser-plasma a été un facteur très important pour le développement d'outils de diagnostics expérimentaux extrêmement performants (indépendamment des modélisations et des simulations numériques sur lesquelles nous allons revenir). On doit citer en particulier les diagnostics de diffusion Thomson sur les ondes de plasma électroniques et acoustiques ioniques, thermiques et cohérentes, avec résolution spatiale, temporelle et spectrale, déflectométrie, qui permettent $d$ 'avoir accès au développement local des ondes longitudinales excitées par les différentes instabilités avec une bonne résolution temporelle.

\section{2 État de l'art actuel}

La théorie et la simulation numérique ont joué un rôle très important dans la conception et l'interprétation des expériences. La modélisation et les simulations numériques n'ont cependant pas encore atteint un niveau où elles seraient en mesure d'évaluer quantitativement les effets de ces instabilités pendant la durée de l'impulsion laser dans un plasma de grande dimension, du type de ceux qui seront mis en jeu dans les expériences de fusion.

Nous avons déjà indiqué à la section précédente les origines de notre incapacité prédictive :

- les mécanismes physiques mis en jeu sont aussi de nature très différente;

- ils sont caractérisés par une très grande disparité d'échelles temporelles et spatiales;

- ils sont couplés entre eux par des boucles de rétroaction;

- lesquelles peuvent être non locales en espace.

Aucun code numérique n'est pour l'instant (et cela restera encore vrai pendant plusieurs années) en mesure de simuler simultanément toutes ces échelles et tous ces mécanismes physiques. Ces traits caractéristiques de l'interaction laserplasma font qu'on ne peut pas espérer obtenir des prédictions quantitatives à 
partir de seules simulations. II est donc absolument indispensable d'effectuer simultanément beaucoup de modélisations, en cherchant à intégrer, d'une façon simplifiée, dans les codes capables de traiter des grands systèmes, les mécanismes physiques qui se développent à des échelles spatiales plus fines.

Ce constat concernant notre faible capacité de prédiction quantitative étant fait, résumons ce qui peut être considéré comme étant bien compris d'une part, et $d$ 'autre part les scénarios de saturation non linéaire qui semblent être actuellement les plus plausibles:

1. les mécanismes linéaires sont maintenant bien compris : effets de longueur finie, effets d'inhomogénéité du plasma, effets de l'incohérence des faisceaux laser ou des fluctuations du plasma. Toutefois, pour faire le lien avec les recommandations qui vont suivre concernant l'enseignement, il y a lieu de dire ici que tous ces mécanismes linéaires représentent maintenant une zoologie extrêmement subtile et complexe, en particulier tout ce qui a rapport aux effets liés à l'inhomogénéité du plasma. Par ailleurs, il est évidemment nécessaire pour interpréter les expériences d'effectuer correctement l'analyse linéaire;

2. les effets non linéaires de type "fluide " sont déjà bien pris en compte : génération d'harmoniques, de sous-harmoniques, modification de l'hydrodynamique provenant du transfert de moment associé aux ondes longitudinales excitées, couplage non linéaire de ces ondes longitudinales entre elles. Ces effets non linéaires de type fluide $n^{\prime}$ apparaissent pas suffisants pour interpréter correctement les expériences actuelles;

3. si l'on compare en effet les résultats expérimentaux avec les prédictions théoriques et les simulations numériques, on peut résumer la situation actuelle (tout du moins pour ce qui concerne les régimes de gain linéaire modéré (quelques unités)) de la façon suivante : les résultats expérimentaux obtenus avec des plasmas de température modérée (de l'ordre du keV) mènent à des réflectivités dues aux instabilités paramétriques (les instabilités de diffusion stimulée Brillouin et Raman) qui sont nettement inférieures aux prédictions théoriques ou numériques. Par ailleurs, les résultats expérimentaux obtenus avec des plasmas chauds (plusieurs keV) mènent au contraire à des réflectivités Raman supérieures aux prédictions théoriques simples;

4. I'interprétation de ces désaccords semble être la suivante :

- la réflectivité globale, bien que petite (de l'ordre de quelques pour cents), provient du développement des instabilités paramétriques dans les grains de tavelure les plus intenses, de sorte qu'elle est déterminée par les mécanismes de saturation non linéaire limitant leur développement dans ces grains de tavelure; 
- il est nécessaire de prendre en compte les effets cinétiques pour décrire l'évolution non linéaire de ces speckles les plus intenses. Le consensus actuel est que les effets cinétiques, dans le cas de l'instabilité Brillouin, réduisent la réflectivité du plasma d'une part en rendant incohérent le couplage entre les ondes transverses et les ondes acoustiques ioniques, et d'autre part en limitant de façon non linéaire le facteur de gain par l'effet d'inhomogénéité spatiale induit par ces effets cinétiques. Dans le cas de l'instabilité Raman, les effets cinétiques peuvent réduire la réflectivité du plasma par des mécanismes semblables à ceux de l'instabilité Brillouin dans le cas d'un plasma de température modérée, ou au contraire augmenter sa réflectivité en permettant des nouveaux modes d'oscillations longitudinales dans le cas de plasmas chauds.

Il y a lieu de signaler ici que les consensus qui ont amené à la mise au point de scénarios de saturation non linéaire des différentes instabilités sont le résultat d'une excellente coordination entre expérimentateurs, théoriciens/modélisateurs, et simulateurs.

\subsection{Domaines à développer : études fondamentales, stratégie de développement de codes de prédiction globale, coordination de l'effort}

La nécessité absolve d'être en mesure de développer des outils prédictifs, vu l'enjeu du problème et l'extrême complexité de la physique de l'interaction laserplasma, requiert un effort très vigoureux. Cet effort devrait se développer selon les trois axes suivants :

- maintien des études fondamentales concernant les mécanismes de saturation non linéaire de la croissance des instabilités paramétriques: modélisation des effets cinétiques dans des codes de type «fluides», prise en compte des non-linéarités des ondes longitudinales excitées par les instabilités Brillouin et Raman, etc.

- stratégie de développement de codes numériques prédictifs via l'analyse mésoscopique : I'analyse mésoscopique consiste à étudier tout $\mathrm{d}^{\prime}$ abord finement les processus physiques se développant sur de courtes échelles spatiales, et à les modéliser ensuite de façon à les prendre en compte de façon simplifiée dans les codes utilisés pour étudier les échelles spatiales supérieures:

- étude des effets cinétiques dans des systèmes petits à l'aide de codes de type PIC a vec collisions, 
- modélisation des effets cinétiques dans des codes de type "fluide»,

- description locale réduite dans laquelle on calcule la réflectivité d'une tranche de plasma de grande taille (mais dans laquelle les paramètres hydrodynamiques varient peu) à l'aide de simulations de type fluide multidimensionnelles. On cherche ensuite à exprimer de façon simple cette réflectivité à l'aide des quelques paramètres macroscopiques caractérisant localement le plasma et le faisceau incident,

- description globale : on calcule la réflectivité globale d'un plasma de grande taille par intégration successive des réflectivités locales calculées en fonction des paramètres macroscopiques caractérisant localement le plasma et le faisceau incident;

- visibilité et coordination de l'effort : la stratégie précédente nécessite donc des activités assez différenciées entre d'une part une recherche de type fondamental consistant en l'analyse et la modélisation d'effets microscopiques et d'autre part en l'intégration de ces modélisations dans un ou plusieurs codes globaux s'approchant de plus en plus d'une description complète. Ces modélisations et ces codes doivent être par ailleurs validés par l'analyse des expériences passées et futures. Au total l'objectif consistant à être en mesure de disposer de codes prédictifs représente donc un effort extrêmement important nécessitant une coopération et une coordination entre les différentes équipes expérimentateursthéoriciens/modélisateurs-simulateurs. Cette coordination doit naturellement $s^{\prime}$ accompagner d'une bonne visibilité et d'une reconnaissance du domaine en tant que tel au sein de la communauté de la fusion par laser.

\subsection{Enseignement}

Nous avons souligné à plusieurs reprises que l'interaction laser-plasma couple de façon non linéaire pratiquement tous les processus physiques propres aux plasmas chauds, depuis l'hydrodynamique jusqu'aux effets cinétiques, en passant par les couplages d'ondes. Ce domaine représente donc une motivation très forte pour que soit dispensé un enseignement solide en physique des plasmas chauds, motivation déjà partagée par l'ensemble de la communauté fusion. Par ailleurs, l'interaction laser-plasma ne doit plus être considérée comme un simple cas particulier d'un cours général sur les couplages d'ondes non linéaires dans les plasmas, tant le sujet s'est maintenant développé en tant que tel, avec toute sa spécificité et sa zoologie d'effets particuliers, que nous avons signalées (instabilités absolves/convectives, effets de longueur finie, $d$ 'inhomogénéité, d'incohérence, couplages non linéaires entre les différentes instabilités, effets cinétiques dans le couplage non linéaire d'ondes, effets non locaux, etc.). Il est donc nécessaire qu'en plus d'un tronc commun solide sur les plasmas 
chauds, soit dispensé un cours spécifique bien construit et doté de suffisamment $d$ 'heures d'enseignement.

\subsection{Coordination et structuration de la communauté}

La communauté française des interactionnistes s'est déjà coordonnée d'ellemême et organise des rencontres biannuelles de "remue-méninges 》 au cours desquelles chaque équipe présente ses travaux aux autres. II y a donc simplement lieu de veiller à ce que cette coordination se maintienne. En revanche, les interactionnistes semblent souffrir d'un manque de reconnaissance de la part du reste de la communauté de la fusion inertielle, sans doute dû à la difficulté du sujet et au manque de formation de base dans ce domaine de cette communauté. II serait donc souhaitable que sous l'égide de l'ILP, par exemple, aient lieu des séminaires d'intérêt général de façon à sensibiliser l'ensemble de la communauté aux enjeux de l'interaction laser-plasma. Des ateliers transcommunautés, seraient aussi sans doute bénéfiques pour l'ensemble des physiciens d'autres disciplines confrontés à des problématiques similaires (génération de courant dans les tokamaks, modification ionosphérique). Enfin, le moment est sans doute venu de chercher à structurer la communauté européenne autour de l'interaction laser-plasma en organisant des écoles d'été de type « Les Houches » sur ce sujet.

\section{2 | Allumage rapide et laser petawatt}

\subsection{Présentation générale}

La France va voir se construire sur son territoire deux équipements scientifiques d'une ampleur et d'une ambition hors norme au plan international. II s'agit d'Iter à Cadarache et du Laser Mégajoule (LMJ) en Aquitaine, tous deux consacrés à comprendre et maîtriser les plasmas chauds thermonucléaires. Programme international civil, Iter confine le combustible par des méthodes magnétiques. Programme de la défense, le LMJ, en cours de construction, qui utilise des lasers pour imploser le combustible et produire de l'énergie par confinement inertiel, va aussi être ouvert au civil. Cette ouverture est déjà une réalité, mise en œuvre dans un premier stade sur le prototype opérationnel du $L M J$, la ligne $d^{\prime}$ intégration laser ou LIL. Elle constitue une formidable opportunité de recherches, génératrice de concepts innovants en science fondamentale, de développements technologiques et d'activités à fortes valeurs ajoutées, ainsi que de perspectives prometteuses dans le domaine de l'énergie du futur. 
La LIL est I'installation prototype qui a pour mission première de valider et qualifier en vraie grandeur la chaîne laser de base du LMJ. Elle est constituée $\mathrm{d}^{\prime}$ une structure laser élémentaire du Laser Mégajoule (LMJ) comportant 8 faisceaux qui, à terme ${ }^{1}$, délivrera en configuration standard une énergie de 60 kilojoules dans l'ultraviolet. La construction et le fonctionnement d'une capacité additionnelle de très grande puissance dite « laser petawatt sur la LIL » qui fait l'objet du projet Petra présenté ici, permettront à la France et l'Europe de développer un programme original et exceptionnel sur le plan international dans le domaine des lasers ultra-intenses et de l'interaction avec la matière irradiée sous très forte densité d'énergie. Les motivations du projet PW-LIL sont principalement de deux ordres:

- mettre à disposition de la communauté scientifique un outil exceptionnel en termes de haute énergie et ultra-haute intensité ;

- lui permettre de réaliser des expériences « intégrées » d'allumage rapide de fusion.

Les spécifications prioritaires du projet impliquent l'addition à la LIL d'un faisceau laser énergétique ultra-intense, émettant des impulsions ultrabrèves 10,5 ps à qq. $10 \mathrm{ps} ; 1$ picoseconde $=10^{-12} \mathrm{~s}$, soit le millionième de millionième de seconde) et énergétique (jusqu'à 3,5 kJ), ce faisceau alimentant l'enceinte expérimentale de la LIL.

Les possibilités technologiques et les avancées des recherches dans le domaine de l'interaction laser-matière permettent aujourd'hui de préciser l'intérêt de disposer d'un faisceau laser d'ultra-haute intensité (UHI) et de haute puissance (PW) auprès de la LIL et du futur LMJ. Une telle installation offrira des possibilités expérimentales sans équivalent dans le monde scientifique. Les études d'interaction d'une impulsion laser UHI avec une cible solide ou gazeuse, aujourd'hui couramment abordées sur les installations 10-100 TW et bientôt envisagées sur les installations de type PW, pourront en effet être étendues aux interactions avec une plus grande variété de cibles, de matériaux et de paramètres, en particulier les plasmas de grandes dimensions produits par les faisceaux de la LIL puis du LMJ, ainsi que les cibles comprimées dans le cadre

\footnotetext{
${ }^{1}$ Elle sera l'installation laser la plus puissante au monde, mis à part le NIF aux États-Unis qui devrait démarrer en même temps. Dans une première phase, quatre faisceaux sont installés dans une configuration regroupée, dite "quadruplet", qui correspond à la chaîne de base du LMJ. Puis dans un second temps, quatre autres faisceaux seront disposés autour de la chambre, en quatre faisceaux indépendants, dits en configuration " éclatée » (ou Octalil). In fine, le quadruplet initial pourra lui aussi être redistribué en configuration éclatée, afin d'obtenir une distribution isotrope des huit faisceaux (directions voisines de celles des diagonales d'un cube) pour laquelle la chambre d'expériences à été préparée. Un quadruplet a été qualifié à la fin de l'année 2004, et les premières expériences d'interaction ont été réalisées.
} 
des études sur la fusion par confinement inertiel (FCI). Les éclairements lumineux supérieurs à $10^{20} \mathrm{~W} / \mathrm{cm}^{2}$ accessibles actuellement sur des surfaces de l'ordre de 10 microns carrés pourront être atteints sur de plus grandes surfaces, ce qui est nécessaire pour la construction de nouvelles sources de rayonnement énergétique (laser $X$, laser gamma $(\gamma$ ), accélérateurs d'électrons et réactions nucléaires) et pour les études sur la matière soumise à des conditions thermodynamiques extrêmes.

L'application fondamentale du couple LIL-Multi-PW concerne « I'allumage rapide » d'une cible de DT précomprimée dans une configuration expérimentale intégrée; ce schéma d'interaction, qui relaxe certaines des contraintes inhérentes aux concepts de $\mathrm{FCl}$ conventionnels à point chaud central (attaques directe et indirecte), est actuellement considéré par la communauté scientifique comme une solution prometteuse pour la production d'énergie par fusion inertielle (EFI).

Les éclairements supérieurs à $10^{21} \mathrm{~W} / \mathrm{cm}^{2}$ qui seront atteints conduiront à l'obtention, au sein de la matière irradiée, de champs électriques mille fois supérieurs à ceux qui maintiennent les électrons au voisinage des noyaux atomiques. Les électrons éjectés seront portés à des vitesses proches de celle de la lumière. Dans de telles conditions, la barrière relativiste sera franchie de plusieurs ordres de grandeur. La théorie quantique relativiste prédit des processus et des mécanismes nouveaux, et donc jamais étudiés en laboratoire, mais supposés exister dans les étoiles jeunes et dans l'Univers.

\subsection{Les enjeux scientifiques}

Bien que ce dossier ait été déjà expertisé en 2003 par un comité scientifique international, nous rappelons brièvement ci-après, par souci d'être complet, les domaines de la physique où le laser MultiPW auprès de la LIL donnera une contribution importante. Le document d'accompagnement en anglais est plus explicite et a une bibliographie à jour.

\subsubsection{Le projet Phare : physique de I'allumage rapide pour l'énergie}

Dans le schéma de base de la $\mathrm{FCl}$, une microbille de deutérium-tritium (DT), est comprimée par la poussée d'un matériau (ablateur), mis en vitesse par la détente d'un plasma éclairé uniformément. Au maximum de compression, un "point chaud" initie les réactions de fusion qui se propagent dans la partie froide et dense du combustible, entrainant un important dégagement d'énergie thermonucléaire. 
Le dimensionnement du Laser Mégajoule a été défini sur la base de ce schéma, avec l'objectif suivant : obtenir un gain de cible de l'ordre de 10 (rapport de l'énergie thermonucléaire dégagée à l'énergie investie) dans un schéma d'implosion par attaque indirecte, consistant à convertir préalablement le rayonnement laser en rayonnement $X$ dans une cavité renfermant la cible sous forme d'un microballon.

L'irradiation directe du microballon par des faisceaux laser est l'autre voie pour comprimer le mélange fusible et obtenir l'allumage par point chaud puis sa combustion. Des mesures conservatoires (essentiellement la disposition des faisceaux autour de la chambre d'interaction) ont été prises dans la conception du LMJ pour pouvoir la mettre en œuvre.

Vis-à-vis des applications énergétiques de la $\mathrm{FCl}$, c'est-à-dire de l'énergie par fusion inertielle (EFI), et compte tenu des difficultés pour produire une compression parfaitement symétrique indispensable pour assurer l'ignition selon les schémas décrits ci-dessus, l'allumage rapide apparaît comme un schéma extrêmement séduisant et prometteur; il consiste à découpler les phases de compression du microballon et d'inflammation du DT et combine impulsions de forte énergie nanosecondes (ns) et de forte intensité picosecondes (ps). Encore faut-il le valider en commençant par les mécanismes de base.

Ce schéma d'allumage rapide soulève donc aujourd'hui des problèmes nouveaux qui nécessitent des recherches approfondies en physique fondamentale :

- la mise en condition de la cible (énergie et degré de symétrie nécessaires) : il s'agit de réaliser les conditions de température et de masse surfacique du cœur comprimé susceptibles d'absorber de manière efficace l'énergie des électrons et ions accélérés par le laser, et d'initier efficacement la combustion;

- la production et la propagation guidée des particules (électrons ou ions) suprathermiques : il faut mieux connaître le rendement de conversion de l'énergie laser en énergie électronique et comprendre la propagation canalisée des particules chargées et le rôle des champs magnétiques ainsi générés, les mécanismes gérant le transport électronique en matière dense et chaude : il s'agit d'étudier la propagation de courants extrêmement intenses, au-dessus de la limite d'Alfven, dans la matière à forte densité et de préciser le rôle des champs électrique et magnétiques sur l'efficacité du transport;

- le dépôt d'énergie par ces particules conduisant à l'allumage : il s'agit de maîtriser la propagation des particules alpha et l'expansion de l'onde de combustion. 
En conclusion, l'association PW-LIL permettra de tester de manière unique ${ }^{2}$ la phénoménologie de l'allumage rapide, soit en géométrie d'attaque directe soit en géométrie d'attaque indirecte, d'étudier (et de quantifier) les phénomènes impliqués dans ce processus.

\subsubsection{Sources secondaires ef diagnostics}

Connaître les plasmas denses et chauds (tels que ceux rencontrés en astrophysique au cœur des étoiles ou lors des expériences de FCl) signifie concrètement avoir accès aux grandeurs thermodynamiques essentielles (température et densité électroniques). II convient pour cela de disposer de sources de rayonnement ou de particules énergétiques qui soient suffisamment brillantes, courtes et spécifiques pour pénétrer dans les plasmas denses, se distinguer du fond de rayonnement et réaliser des mesures instantanées.

Les études menées sur les installations laser multiterawatt (ps et subps) actuelles montrent qu'on sait produire de façon efficace :

- des rayons $\mathrm{X}$ dur et $\gamma$ (du keV à plusieurs dizaines de $\mathrm{MeV}$ );

- des lasers à rayons $X$ (dans la gamme 5-25 nanomètres (nm)) ;

- des faisceaux d'électrons relativistes de courant très élevé (jusqu'à $10^{14} \mathrm{~A} / \mathrm{cm}^{2}$ );

- des faisceaux de neutrons, positrons, protons et ions lourds d'énergie audelà du MeV.

Les techniques associées : radiographie, imagerie, interférométrie, diffusion Thomson sont connues depuis de nombreuses années dans le domaine optique. Substituer aux sources correspondantes des sources plus pénétrantes, plus brillantes et de plus faible durée, telles que celles générées, directement ou indirectement, par l'interaction laser PW-matière, améliorera sensiblement les performances des diagnostics et donnera accès à une meilleure compréhension de la matière dans des conditions " extrêmes ". Les développements récents en radiographie par protons et la mesure des champs électriques internes au plasma par déflection de protons et d'ions légers sont particulièrement importants pour les diagnostics de cible comprimés. Trois caractéristiques uniques des rayonnement et des particules produites par un laser PW sont particulièrement intéressantes pour le diagnostic des plasmas denses et chauds : les sources seront plus énergétiques (photons plus "durs 》 particules plus énergétiques, et donc plus

\footnotetext{
${ }^{2}$ En dehors des États-Unis.
} 
pénétrantes), les sources seront plus brillantes et plus intenses que le rayonnement propre de la matière étudiée, la durée de l'émission sera plus brève que les temps caractéristiques d'expansion du plasma, supprimant ainsi l'effet de «flou ».

Les connaissances, tant théoriques qu'expérimentales, acquises ces dernières années, permettent de dimensionner et de caractériser ces sources de rayonnement et de particules à l'échelle des 100 TW. Le changement d'échelle au niveau du petawatt reste encore à être validé. Mais, outre l'intérêt scientifique explicité plus haut d'un tel faisceau PW sur la ligne d'intégration laser (LIL), cette approche devrait s'avérer primordiale à terme pour les futures expériences scientifiques sur le LMJ.

\subsubsection{Matière à haute densité d'énergie}

L'obtention de "grands " milieux à la fois denses et chauds (ou tièdes) sera une caractéristique unique du tandem PW-LIL. La physique abordée dans ce domaine est, bien sûr, corrélée avec les précédents avec des retombées dans $\mathrm{d}^{\prime}$ autres domaines scientifiques comme la géophysique, les sciences de la Terre et des planètes telluriques, et l'astrophysique. Les défis présentés par ces études se concentrent dans la production d'états de la matière inédits de manière parfaitement contrôlée. Au plan expérimental, c'est encore la conjonction d'un laser en impulsions longues (LIL) avec un laser picoseconde (Multi-PW) qui est le point crucial. Les domaines suivants seront plus particulièrement étudiés :

- les propriétés thermodynamiques de la matière dense à basse (10 eV) ef haute ( $1 \mathrm{keV}$ et plus) température : il s'agit des études d'équation d'état des matériaux d'intérêt pour l'EFI et pour la physique des planètes, et de l'étude des instabilités hydrodynamiques qui pourront être abordées avec de nouveaux moyens incluant la radiographie avec des particules chargées ;

- l'étude de l'émission (émissivité) et l'absorption (opacité) des plasmas dans des conditions thermodynamiques extrêmes de la matière comprimée ;

- la cinétique atomique hors d'équilibre : il s'agit d'étudier les effets instationnaires sur la dynamique d'ionisation. En effet, avec un laser PW, la durée de l'impulsion est plus brève que les temps d'expansion hydrodynamique mais comparable aux cinétiques $d^{\prime}$ ionisation ;

- la génération et la mise en œuvre de sources secondaires très pénétrantes telles que les sources d'électrons rapides ou d'ions légers rapide : les premières études devraient aborder l'étude du chauffage isochorique des cibles afin d'obtenir des températures élevées tout en maintenant une forte 
densité. De même, des sources secondaires de particules seront utilisées pour caractériser le pouvoir d'arrêt des électrons et des ions dans la matière comprimée. Pour ce dernier point, il s'agira de mesures tout à fait complémentaires de ce qui pourra se faire dans une installation telle que GSI-Darmstadt où un laser 1 PW sera couplé à un accélérateur d'ions lourds.

\subsubsection{Matière portée à des conditions extrêmes}

L'accès, grâce au laser PW, à des domaines " frontières de la physique 》 sera un vecteur $d^{\prime}$ ouverture vers d'autres communautés scientifiques, surtout celles qui $n$ 'ont pas accès aux « manipulations 》 expérimentales en raison de l'inaccessibilité de leur objet d'étude (galaxies ou autres objets astrophysiques) ou en raison de la faiblesse des forces mises en jeu (la gravitation par exemple). En effet, avec le laser Multi-PW on pourra accéder à des densités de puissance records, supérieures à $10^{21} \mathrm{~W} / \mathrm{cm}^{2}$, correspondant à des énergies d'oscillation des électrons qui sont nettement du domaine relativiste ou ultra-relativiste. La matière est alors soumise à des conditions extrêmes dont des manifestations sont :

- la génération de champs magnétiques extrêmement élevés supérieurs à $10^{9} \mathrm{G}$ similaires à ceux qui doivent participer à l'évolution des naines blanches et des étoiles à neutrons. Des champs magnétiques de cette intensité ne peuvent pas être produits autrement en laboratoire et devraient attirer des communautés autres que celles gravitant autour de la physique des plasmas;

- des pressions très fortes (plusieurs Gbar), similaires à celles rencontrées aux confins des trous noirs, sièges d'accélération très importante $\left(10^{24} \mathrm{G}\right)$. $\mathrm{Ce}$ thème $\mathrm{d}^{\prime}$ exploration se situe réellement à la frontière de la physique des plasmas, de l'électrodynamique quantique, de la relativité générale et de la physique des particules élémentaires.

\subsubsection{Autres retombées scientifiques}

L'apparition de nouveaux instruments entraîne toujours de nouvelles idées, de nouvelles applications. Des communautés scientifiques autres que celles du premier cercle des lasers intenses et plasmas ont participé aux réflexions prospectives sur l'utilisation scientifique du LMJ. Il a été ainsi noté que les états de la matière produits par interaction combinée des impulsions nanosecondes (LIL) et picosecondes (PW) sont très exotiques du point de vue des différents domaines de physique abordables. On en donne trois exemples ci-dessous (liste non exclusive). 
Pour la physique de la matière condensée l'attractivité de l'association LIL-PW est liée à l'exploration du diagramme thermodynamique hors des chemins usuels (lors de la détente adiabatique d'un plasma créé par interaction laser-solide à la densité du solide et à des températures du keV).

Dans le domaine de la physique nucléaire, la matière, portée à l'état de plasma dense, peut être le siège de réactions de fission et de fusion qui génèrent un nombre important de particules (ions et électrons suprathermiques, neutrons et photons). Ces dernières vont interagir avec leur milieu et le modifier. Ces phénomènes, tels que, par exemple, le processus NEET (Nuclear Excitation by Electronic Transition), peuvent être reproduits en laboratoire lorsque des particules analogues, créées par interaction laser PW-solide, sont « injectées » dans un plasma précomprimé. Du fait des volumes macroscopiques de plasma produits par le laser Multi-PW et de la forte brillance des sources secondaires de particules générées, l'étude des faibles sections efficaces de réaction sera enfin à notre portée, en physique subatomique.

Dans le domaine de l'astrophysique on peut modéliser à l'échelle du laboratoire les processus prévus dans les étoiles et le milieu interstellaire : instabilités des fronts de chocs, accélération des particules dans les champs aléatoires, transition de phases dans le centre des étoiles, connaissance de l'opacité de divers matériaux, conditions thermodynamiques " exotiques » (quelques $\mathrm{g} / \mathrm{cm}^{3}$, quelques $\mathrm{keV}$ ), etc.

\subsection{Stratégie régionale, nationale et européenne}

\subsubsection{Un environnement très compétitif}

Des lasers petawatts de haute énergie sont construits ou en projet dans de nombreux endroits de par le monde, au CLF du Rutherford Appleton Laboratory en Angleterre, au GSI Darmstadt, ailleurs en Europe (PALs à Prague, à Jena, au LOA à Palaiseau), aux États-Unis (Livermore, Rochester, AnnArbor, Sandia, etc.). Le Lawrence Livermore National Laboratory et I'université de Rochester planifient des additions petawatt à leurs installations respectives, NIF et Omega. Au Japon, en préparation du projet FIREX consacré à la démonstration de l'allumage rapide, un laser petawatt a été déjà couplé à l'installation Gekko 12.

C'est dans ce contexte extrêmement compétitif que la combinaison des impulsions très énergétiques de la LIL avec les impulsions du laser petawatt rendront cette installation ambitieuse absolument unique en Europe et au premier rang mondial avec ses concurrents aux États-Unis et au Japon. 


\subsubsection{La situation française}

Du fait du niveau d'investissement relativement élevé de ce type d'installation laser, une coordination s'est progressivement mise en place, depuis bientôt une dizaine d'années, entre les grands organismes de la recherche française impliqués dans ce domaine : le CEA et le CNRS. Elle a donné lieu, jusqu'en 1999, à des collaborations expérimentales sur les installations du CEA à Limeil Brévannes, en particulier avec le laser pionnier en matière de laser " petawatt», le laser P102. Jusqu'à récemment, la plupart des physiciens des plasmas chauds par laser se situent en Île-de-France, se partageant principalement entre l'École polytechnique (Luli et CPHT) et son groupement d'utilisateurs académiques, et la DIF au CEA-Bruyères-le-Châtel.

Le CNRS et le CEA ont décidé en 1998 de joindre leurs efforts avec l'École polytechnique (et la DGA) dans l'opération de Jouvence du Luli (projet Luli 2000). Ce programme comporte un volet de chaîne " petawatt ", dit programme Pico 2000 au niveau de 400 joules en $400 \mathrm{fs}$, laser couplé à un ou des faisceaux nanoseconde et kilojoule. L'ensemble couplé nanoseconde et petawatt sera opérationnel au cours de l'année 2007. Remarquons cependant qu'il s'agit d'une technologie ancienne qui limite l'énergie émise à $0,5 \mathrm{~kJ}$, soit pratiquement un ordre de grandeur de moins par rapport à ce que pourra produire le laser PW sur la LIL.

On doit aussi mentionner, dans la classe des lasers à base de titane saphir, plusieurs installations fonctionnent actuellement en régime d'impulsions ultrabrèves (de 20 à $100 \mathrm{fs}$ ), avec une puissance de quelques dizaines de terawatt, au Laboratoire d'optique appliquée à Palaiseau et au CEA-Saclay par exemple. Le LOA affiche cependant depuis peu un projet extrêmement ambitieux défendu auprès de l'ESFRI, dit ELI pour Extreme Light Infrastructure, de passage au niveau de la fraction d'exawatt en régime d'impulsions très courtes (jusqu'à $2 \mathrm{~kJ}$ en $15 \mathrm{fs}$ à une longueur d'onde de $2 \mu \mathrm{m}$ ).

En conclusion, on peut noter que les équipes académiques françaises sont par tradition très actives dans ce domaine. Une des origines de cette situation réside dans les programmes du CEA/Dam qui ont engendré des besoins continus de formation et de support amont en physique des plasmas chauds. Actuellement, on note que cette communauté se révèle particulièrement dynamique, ce qui se traduit par une progressions dans des outils de grande qualité et très onéreux, mais paradoxalement, par aussi un vieillissement de la population. Les nouveaux besoins, suite au programme LMJ, ne font que qu'accentuer cette nécessité de renforcer la communauté, par exemple avec l'UMR de Bordeaux. La coordination et structuration des activités des installations et populations scientifiques des 
pôles existants (le francilien) et en construction (l'aquitain) ont justifié la création de l'Institut laser plasma (ILP).

\subsubsection{La situation européenne}

En Europe, et en dehors de la France, on trouve deux projets de la classe petawatt reposant sur la technologie verre-néodyme, sous la responsabilité du Rutherford Appleton Laboratory (RAL) et du Gesellschaft für Schwerionen (GSI) à Darmstadt. Ces deux programmes reposent sur la récupération des chaînes amplificatrices des lasers Nova et Phebus. Ces lasers sont donc limités, de par leur technologie et comme l'a été le premier laser PW sur Nova, à des énergies subkilojoule.

La chaîne PW sur Vulcan au RAL est dans sa phase de première exploitation, alors que le laser PW de GSI est construit parallèlement à celui du Luli. Cependant, contrairement à LULI2000, le laser petawatt du RAL n'est couplé à aucun autre faisceau de haute énergie alors que celui du GSI le sera avec un faisceau d'ions lourds. La justification des ces programmes est l'allumage rapide des cibles thermonucléaires, avec des lasers pour le RAL ou des ions lourds pour le GSI.

En conclusion, on doit noter que les programmes européens, TMR puis IHP, et maintenant du $6^{\mathrm{e}} \mathrm{PCRD}$, ont permis à la communauté des scientifiques européens de collaborer activement dans le domaine des plasmas chauds créés par laser et d'initier de nouvelles recherches dans des champs connexes, que ce soit en astrophysique, géophysique, matériaux sous chocs, etc.

Il faut cependant mettre en avant le dossier qui porte toute une communauté en Europe, qui est le projet HiPER d'installation dévolue à la démonstration en grandeur réelle de l'allumage rapide. II s'agit de coupler un laser de $200 \mathrm{~kJ}$ en régime nanoseconde avec des lignes petawatts totalisant de l'ordre de $60 \mathrm{~kJ}$ en quelques picosecondes de durée. Le projet Petra (PW-LIL) se positionne donc comme un démonstrateur scientifique et technologique incontournable d'une roadmap européenne où se positionnerait en phase terminale une expérience d'ignition.

\subsubsection{La situation internationale hors de l'Europe}

Hors Europe, on trouve des programmes majeurs de laser petawatt, ciblé majoritairement pour l'allumage rapide, dans deux pays, le Japon et les USA, en attendant la Chine. 
Le programme du Japon

Au Japon à l'ILE de l'université d'Osaka, un programme de ligne petawatt reposant sur les principes démontrés à Livemore sur Nova, et le même que celui mis en application au RAL, est désormais opérationnel. Il produit une impulsion limitée à 0,5 petawatt, mais est couplé aux douze faisceaux du laser à haute énergie (quelques dizaines de kilojoules)

Les études se consacrent majoritairement sur l'allumage rapide, vu la mission de ce laboratoire qui est l'énergie par fusion inertielle. Des expériences récentes réalisées par un consortium regroupant des scientifiques des États-Unis, du Royaume-Uni et du Japon ont démontré un effet de renforcement d'un facteur 10 sur la production des neutrons par l'utilisation de ce laser.

II s'agit pour l'instant de l'étape de démonstration en vue de faire approuver le projet FIREX (pour Fast Ignition Realization Experiment) dont l'objectif est la démonstration de l'allumage rapide. La première phase de ce projet qui est en cours de construction et qui serait mis en service à partir de fin 2007, consiste en un laser de $10 \mathrm{~kJ}$ en 10. La deuxième phase, FIREX2, consiste à passer du couplage à Gekko 12 à un couplage avec un laser à 92 faisceaux de façon à obtenir une compression à $\rho \mathrm{R}=0,8 \mathrm{gcm}^{2}$ et une démonstration de l'autoallumage et du « break-even » pour 2013.

\section{Le programme petawatt aux États-Unis}

De nombreux projets petawatt existent actuellement aux États-Unis : University of Nevada, Sandia (machine Z-pinch) à partir du Beamlet qui était le prototype du NIF, laboratoire de General Atomics à San Diego. Le programme majeur financé qui s'apparent à celui du PW sur la LIL est celui du Laboratory for Laser Energetics à Rochester (LLE), qui repose sur les mêmes technologies que le LMJ.

\section{Projet Omega EP}

Le projet Omega EP de Rochester (EP pour Extended Performance), repose sur le laser Omega proprement dit qui fonctionne avec ses 60 faisceaux de 0,5 kJ, soit un total de $30 \mathrm{~kJ}$ à $0,35 \mu \mathrm{m}$. Il consiste en l'extension des capacités de cette installations par des impulsions courtes PW obtenus grâce à l'adjonction de faisceaux du type NIF, donc similaires à ceux de la LIL et du LMJ. Néanmoins, si ce programme s'apparente de très près à celui du PW sur la LIL, il en diffère. Par exemple, n'ayant pas à faire les investissements pour les maintenances des équipements du NIF, Rochester a préféré développer une nouvelle conception de 
la mécanique avec des faisceaux indépendants sur rails posés au sol. Bien qu'il soit intéressant à considérer pour la LIL, ce schéma n'est directement applicable sur la LIL. La construction doit s'étager jusqu'à fin 2007. Notons que le projet OMEGAEP se veut aussi être un démonstrateur technologique pour des chaînes multi-petawatt sur le NIF. 
